\title{
Rare presentation of Takayasu's aortoarteritis after double valve replacement
}

\author{
Shivanand Gangahanumaiah, MCh, ${ }^{a}$ Vijayakumar Raju, $\mathrm{MS}^{\text {a }}$ Ram Kumar Jayavelan, $\mathrm{MCh},{ }^{\mathrm{a}}$ Alpha Mathew Kavunkal, $\mathrm{MCh},{ }^{\mathrm{a}}$ \\ Vijit Koshy Cherian, MCh, ${ }^{a}$ Debashish Danda, DM, ${ }^{\text {b }}$ and Velayudhan V. Bashi, MCh, ${ }^{\mathrm{C}}$ Vellore and Chennai, India
}

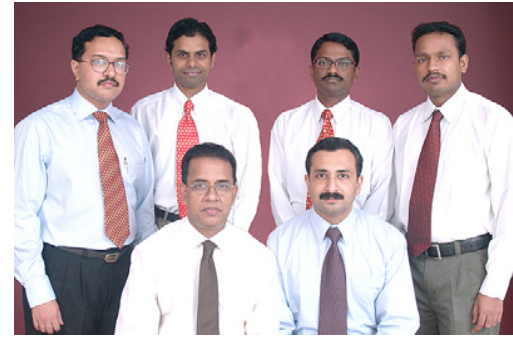

Drs Kavunkal, Ramkumar, Ganguhanumaiah, and Raju (standing, left to right); Drs Bashi and Cherian (seated, left to right)

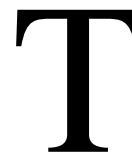

akayasu's aortoarteritis is a chronic inflammatory disease involving the aorta, arteries arising from the aorta, and, frequently, the pulmonary arteries. The inflammation leads to either stenosis and occlusion of the involved artery, aneurysm formation, or both. ${ }^{1}$ We report a rare case of evolving Takayasu's aortoarteritis diagnosed after double valve replacement. A similar presentation has not been reported earlier.

\section{Clinical Summary}

A 29-year-old woman presented with dyspnea on exertion that progressed to New York Heart Association class IV over a period of 1 month. She gave no history suggestive of rheumatic fever. Echocardiographic analysis revealed severe mitral regurgitation, moderate aortic regurgitation, and pulmonary hypertension with good left ventricular function. She presented with features of congestive cardiac failure. She was hospitalized on 2 previous occasions for congestive cardiac failure. The C-reactive protein level was $121 \mathrm{mg} / \mathrm{L}$, the antistreptolysin-O titer was $250 \mathrm{IU} / \mathrm{mL}$, and the erythrocyte sedimentation rate was $35 \mathrm{~mm} / \mathrm{h}$. Chest roentgenographic study showed cardiomegaly (cardiothoracic ratio of $62 \%$ ) with biatrial enlargement and pulmonary venous hypertension. Investigations for other inflammatory markers, such as circulating antineutrophil cytoplasmic antibody (C-ANCA), perinuclear P-ANCA, and antinuclear an-

From the Departments of Cardiothoracic Surgery ${ }^{a}$ and Rheumatology, Christian Medical College and Hospital, Vellore, India; and the Department of Cardiothoracic Surgery, ${ }^{c}$ MIOT Hospital, Chennai, India.

Received for publication Aug 31, 2007; accepted for publication Oct 19, 2007.

Address for reprints: Shivanand Gangahanumaiah, MCh, Department of Cardiothoracic Surgery, Christian Medical College and Hospital, Vellore, Tamil Nadu 632004, India (E-mail: cardiothoracic1@cmcvellore.ac.in).

J Thorac Cardiovasc Surg 2008;135:440-1

$0022-5223 / \$ 34.00$

Copyright (C) 2008 by The American Association for Thoracic Surgery doi:10.1016/j.jtcvs.2007.10.031 tibody (ANA), were negative. She was started on intravenous frusemide and oral aspirin, despite which she continued to be in refractory cardiac failure. In view of her symptoms, she was sent for early aortic and mitral valve replacement.

We performed the operation through a midline sternotomy. The heart was moderately enlarged, with evidence of pericarditis. The ascending aorta measured about $3 \mathrm{~cm}$ in diameter but appeared to be inflamed. The unusual feature noticed at surgical intervention was that the aortic wall was thickened. Thickened aortic walls are not unusual in many patients with disease of rheumatic cause who we see in this subcontinent. The coronary ostia were normal. The aortic and mitral valves appeared myxoid, with poor coaptation of the mitral leaflets. Both valves were excised and replaced with mechanical prosthetic valves. Cardiopulmonary bypass was stopped without difficulty, and the patient had an uneventful immediate postoperative recovery. Aortic and mitral valve biopsy revealed extensive myxomatous deposits in the spongiosa of the valve and hyalinization of the fibrosa. There were focal clusters of mononuclear inflammatory cells with vascularization. Aschoff granulomas were not present.

Six months later, she presented with complaints of fatigue and dyspnea on exertion of New York Heart Association class II. All the peripheral pulses were well felt and equal. Prosthetic valve sounds were well heard. Transesophageal echocardiographic analysis revealed a dilated ascending aorta. A 64-slice computed tomographic scan revealed a large fusiform aneurysm measuring 9.7 $\mathrm{cm}$ in length and involving the ascending aorta. The distal end of the aneurysm was seen just proximal to the innominate artery (Figure 1). No dissection or thrombus was identified. The aortic arch and its branches were normal. Replacement of the ascending aorta was planned.

The femoral artery and vein were cannulated, and she was started on cardiopulmonary bypass. The chest was opened through the previous sternotomy incision. Ostial cardioplegia was instituted. The previous aortic prosthetic valve was found to be hanging precariously on a degenerative aortic root, and hence a redo Bentall procedure was indicated. The coronary ostia were normal. After excising the aortic root, a valved conduit was seated. Coronary buttons were attached to the conduit. Total circulatory arrest at $16^{\circ} \mathrm{C}$ was established. The distal part of the diseased ascending aorta was excised, and the conduit was anastomosed distally to the aorta at the origin of the innominate artery. The heart picked up spontaneously in sinus rhythm. She was extubated the next day. On the 14th postoperative day, she went into complete heart block, for which a permanent pacemaker was inserted.

Biopsy of the aortic root showed granulomatous inflammation and myxomatous change suggestive of aortoarteritis. Repeat computed tomographic angiographic analysis revealed minimal outpouchings of contrast seen in relation to the aortic arch (Figure 2). There was diffuse circumferential wall thickening of the left 


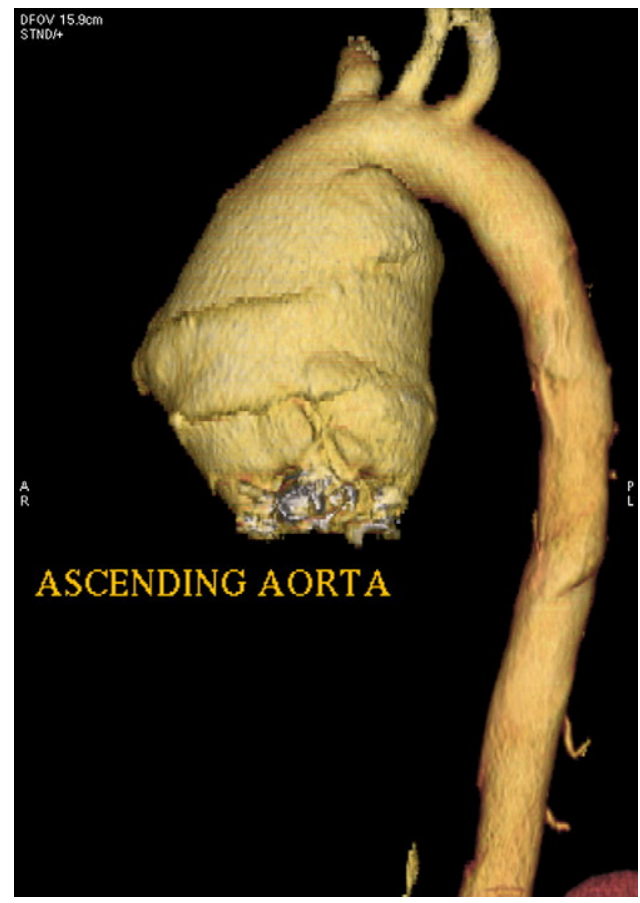

Figure 1. Computed tomographic reconstruction of the ascending aortic aneurysm with valve in situ.

common carotid artery with narrowing of the lumen and also minimal wall thickening of the right brachiocephalic and right common carotid arteries. The left subclavian artery showed poor flow and mild aneurysmal dilatation with thrombus. The descending aorta and the visualized abdominal aorta, with its major branches, appeared normal.

Based on the age group, histologic features, and computed tomographic angiographic analysis with nonreactive VDRL (Venereal Disease Research Laboratory) and negative C-ANCA, negative P-ANCA, and negative ANA results, a diagnosis of type IIA Takayasu's arteritis was made.

She was started on steroids and immunosuppressants. Review at 6 months revealed she had controlled disease, with inflammatory markers minimally increased.

\section{Discussion}

Occlusive Takayasu's arteriopathy has been considered essentially one of the occlusive arterial diseases of the aorta, its main branches, and the pulmonary artery. However, narrowing, occlusion, or both are sometimes combined with aneurysm, as well as dilatation. In this disease process occlusive lesions can be first produced, and in some cases aneurysmal changes follow at any other portion of the aorta and its main branches. ${ }^{1}$

Many criteria have been proposed in the past to clinically diagnose this condition; however, the American College of Rheumatology criteria have been reported to have a sensitivity of $90.5 \%$ and a specificity of $97.8 \% .^{2}$ The patient described above did not fulfill the American College of Rheumatology clinical criteria to be given a preoperative diagnosis of Takayasu's arteritis.

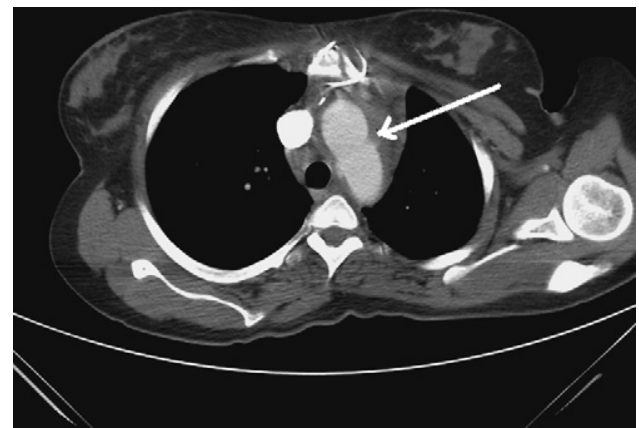

Figure 2. Computed tomographic scan showing minimal outpouchings of contrast seen in relation to the aortic arch.

When Takayasu's arteritis is diagnosed, the treatment begins with pharmacologic control of the acute arteritis to induce clinical remission. Surgical treatment is needed when complications of stenosis or occlusion or, less frequently, an aneurysm develops similarly to patients affected by other diseases. Surgical intervention should be performed when the patient is in remission to avoid complications caused by inflammation, such as restenosis, anastomotic failure, thrombosis, hemorrhage, and infection. ${ }^{3}$ When our patient presented primarily with features of severe mitral regurgitation and moderate aortic regurgitation, there were no features of stenosis, occlusion, or aneurysm formation. However, her inflammatory markers were increased. It was shown by Sandring and Welin, in $1961,{ }^{4}$ that a high antistreptolysin-O titer can be found in some of the patients with Takayasu's arteritis. The refractory congestive cardiac failure in our patient led to an earlier operation.

The patient had degenerative dilatation of the aortic root and a massive aneurysm of the ascending aorta after double valve replacement as a manifestation of the aortoarteritis. Such a presentation has not been reported earlier.

A diagnosis of Takayasu's arteritis was made retrospectively with the histopathologic examination of the resected aortic wall and the computed tomographic angiography. Angiography is the gold standard for evaluation of vascular lesions. Angiography allows a topographic classification, which correlates anatomic involvement, clinical manifestation, and prognosis. ${ }^{5}$

Although primary diagnosis of Takayasu's aortoarteritis was difficult in this patient because of atypical presentation, a possibility of Takayasu's aortoarteritis should be kept in mind in young women with increased inflammatory markers and antistreptolysin-O titers.

\section{References}

1. Talwar KK, Seth S. Cardiac manifestations of Nonspecific aortoarteritis. In: Panja M, editor. Nonspecific aortoarteritis. Kolkata, India: Marksman Media Services; 2001. p. 61-7.

2. Arend WP, Michel BA, Bloch DA, et al. The ACR 1990 Criteria for the classification of Takayasu's arteritis. Arthritis Rheum. 1990;33:1129-34.

3. Maffei S, Di Renzo M, Bova G, Auteri A, Pasqui AL. Takayasu'a arteritis: a review of the literature. Intern Emerg Med. 2006;1:105-12.

4. Sandering H, Welin G. Aortic arch syndrome with special reference to Rheumatoid arteritis. Acta Med Scand. 1961;170:1-19.

5. Numano F. Differences in clinical presentation and outcome in different countries for Takayasu's arteritis. Curr Opin Rheumatol. 1997;9:12-5. 